\title{
Human Rabies Still Exists in Urban and Peri-Urban Areas in Mali-National Data-Base Analysis of the Capital of Mali from 2007 to 2017
}

\author{
Abdel Kader Traoré1,2,3,4, Assétou Soukho Kaya1, Ousmane Koné2,4,5, Youssouf Fofana2,6, \\ Mamadou Cissoko',2, Zakaria Kéïta' ${ }^{2}$, Makan Fofana ${ }^{2,7}$, Aïchatou Margueritte Diarra ${ }^{8}$ \\ ${ }^{1}$ Department of Internal Medicine, Universitary Hospital of Point G, Bamako, Mali \\ ${ }^{2}$ RIWA/REAO/MALI, Rabies in West Africa (RIWA), Mali \\ ${ }^{3}$ RIWA/REAO, INTERNATIONAL, Rabies in West Africa (RIWA), Mali \\ ${ }^{4}$ PARACON, Pan-African Rabies Control Network, Bamako, Mali \\ ${ }^{5}$ Service of Epidemiology-Department of Comunotary Health, National Institute of Public Health, INRSP, Bamako, Mali \\ ${ }^{6}$ Department of Internal Medicine, University Hospital Mother-Child Luxembourg, Bamako, Mali \\ ${ }^{7}$ Disease Prevention and Control Division, National Directorate of Health, Ministry of Health and Social Affairs, Bamako, Mali \\ ${ }^{8}$ Management of Small and Medium Enterprises, Bamako, Mali \\ Email: traoreak@gmail.com
}

How to cite this paper: Traoré, A.K., Kaya, A.S., Koné, O., Fofana, Y., Cissoko, M., Kéita, Z., Fofana, M. and Diarra, A.M. (2019) Human Rabies Still Exists in Urban and Peri-Urban Areas in Mali-National Data-Base Analysis of the Capital of Mali from 2007 to 2017. Open Journal of Internal Medicine, 9, 19-25.

https://doi.org/10.4236/ojim.2019.92004

Received: May 11, 2019

Accepted: June 24, 2019

Published: June 27, 2019

Copyright $\odot 2019$ by author(s) and Scientific Research Publishing Inc. This work is licensed under the Creative Commons Attribution International License (CC BY 4.0).

http://creativecommons.org/licenses/by/4.0/

\begin{abstract}
From 2007 to 2017 sixty-three (63) new cases of human rabies have been identified in Mali on an estimated total population in 2017 of 17,650,195 inhabitants. This is an incidence of 0.32 cases for 100,000 inhabitants. All these cases occurred in urban areas and involved the two sexes. The age group most affected was the one less than 20 years old. The majority of the victims (73.1\%) were pre-schools, school-aged pupils and housewives. In $97 \%$ of the cases, the animal in question was a dog and the type of exposure of the bite. Eighty-two percent (82\%) of the victims had received no local medical treatment or the post exposure quasi prophylaxis treatment. The median duration between the first symptoms and death among the cases of human rabies was 3 to 7 days. The median duration between the occurrence of the first signs and death was four (4) days. No virological confirmation had been conducted on the patients. The rage disease is retained as a priority disease, since the adoption of the strategy of "integrated monitoring of the disease and response" in 2008. However, in spite of the efforts made, progress still remains to be effected to assess the actual impact of rabies in Mali.
\end{abstract}

\section{Keywords}

Rabies, Urban Area, Mali 


\section{Introduction}

Rabies is a zoonosis accidentally transmitted to man which, once declared, is constantly threatening. In fact, despite the existence of very effective vaccines for human and veterinary use, it remains a major public health problem, with 55,000 deaths in the world each year, of which 99\% occur in Africa and Asia. It is the tenth $\left(10^{\text {th }}\right)$ cause of death by infection in humans. Africa is paying a heavy price for this disease, with 26,000 fatal cases annually, according to the recent estimates of the World Health Organization (WHO) [1]. Each year, approximately 12 million people receive treatment after exposure to animals in which rabies is suspected to reside [2].

It is a cosmopolitan affection which produced a range of meningoencephalitis cases with fatal outcome of the etiological agents that are grouped within the Lyssavirus genus. Vaccination in pre-exposure or prophylaxis post-exposure (PPP) is the only effective measures against this disease. After exposure, the rabies vaccine should be administered as soon as possible following the protocols recommended by the WHO with, hopefully, a success rate of close to $100 \%$. In Western countries, human rabies has been eliminated thanks to the Pasteurian treatment. This disease is an endemical epidemic to be fought in the developing countries considered as regions at high risk due to the existence of the enzootic or incursion zones of canine rabies.

In Mali the rage disease is retained as a priority disease since the adoption of the strategy of "integrated monitoring of the disease and response" in 2008, but this integration is not yet effective.

Our goal in this work is to do an analysis of the epidemiological aspects of new human rabies cases in the Bamako district from 2007 to 2017.

\section{Goals}

\subsection{General Goal}

To describe the epidemiological profile of new human rabies cases in the Bamako district for the period between January 1, 2007 and 31 December 2017.

\subsection{Specific Goals}

- Determine the incidence of human rabies cases;

- Describe the socio-demographic characteristics and clinical trials of persons infected with human rabies;

- Describe the animals complained of and the type of exposure to rabies in the District of Bamako;

- Make suggestions for improvement of the monitoring of human rabies cases.

\section{Methodology}

\subsection{Framework of the Study}

The study was done on the basis of the national database, implemented in the 
prevention division to combat the disease (DPLM) of the Mali National Directorate of Health (DNS).

Located in the neighborhood of the river, the rabies center is in cohabitation with the national vaccination center. This is the national reference center for the support and monitoring of the rage disease in Mali. The cases of human rabies are referred before being transferred to the service of infectious and tropical diseases of the $\mathrm{CHU}$ of Point $\mathrm{G}$.

\subsection{Type of Study and Duration of the Study}

The study consisted of a transversal study with a retrospective screening of data from January 2007 to December 2017.

\subsection{Population Sample Studied}

The population sample studied was composed of people of all ages and of both sexes, from Bamako, exposed to the risk of rabies infection by contact, bite, scratch or licking on mucous membranes or skin eroded by a warm-blooded animal, domestic or wild, presenting obvious signs or not of the rage disease.

\subsection{Inclusion Criteria}

We analyzed the records of the patients received at the DPLM and we have included the records of patients who met the definition of cases of human rabies.

\section{Definition of cases of human rabies}

We have considered as cases of human rabies any deceased person in Table 1 and Table 2 of neuropsychiatric signs, with hydrophobia, following an infectious contact (a bite or a scratch biting on a mucous membrane or eroded skin) by a disappeared or death animal or an animal of which the biological diagnosis of rabies was confirmed.

\subsection{Data Gathering}

Data on characteristics of the persons assaulted by animals were collected from registers kept by the Center dealing with the rabies disease.

The information collected was related:

- To the socio-demographic characteristics of the patients (gender, age, profession, place of residence, contact);

- On the reasons of medical consultation (bite, scratch, or licking and the circumstances of the incident);

- To the description of the injuries (place, aspect and date of the incident);

- The gender and the origin of the animal in question;

- The treatment received (treatment of lesions, anti-rabies vaccine and other treatment received);

- To the veterinary supervision of the animal in question (number and date of the veterinary certificates issued, decision of continuation or cessation of the anti-rabies vaccine by the patient; 
- To the evolution of the patient until death occurred.

\subsection{Data Analysis}

The data analysis was carried out by the Epi Info software version.

\section{Results (Tables 1-3)}

Table 1. Distribution of cases of human rabies in Bamako from 2007 to 2017.

\begin{tabular}{ccccccccccccccc}
\hline & \multicolumn{10}{c}{ Year } \\
\cline { 2 - 12 } & 2007 & 2008 & 2009 & 2010 & 2011 & 2012 & 2013 & 2014 & 2015 & 2016 & 2017 & Total \\
\hline Staff & 9 & 6 & 8 & 5 & 3 & 9 & 5 & 1 & 2 & 8 & 7 & 63 \\
Percentage & $14.3 \%$ & $9.6 \%$ & $12.7 \%$ & $7.9 \%$ & $4.8 \%$ & $14.2 \%$ & $7.9 \%$ & $1.6 \%$ & $3.2 \%$ & $12.7 \%$ & $11.1 \%$ & $100 \%$ \\
\hline
\end{tabular}

Table 2. Distribution of cases of human rabies according to gender.

\begin{tabular}{cccccccccccccc}
\hline & \multicolumn{10}{c}{ Year } \\
\cline { 2 - 13 }$y$ & 2007 & 2008 & 2009 & 2010 & 2011 & 2012 & 2013 & 2014 & 2015 & 2016 & 2017 & Total \\
\hline Male & 6 & 4 & 7 & 4 & 3 & 6 & 4 & 1 & 2 & 5 & 5 & 47 \\
Female & 3 & 2 & 1 & 1 & 0 & 3 & 1 & 0 & 0 & 3 & 2 & 16 \\
Total & 9 & 6 & 8 & 5 & 3 & 9 & 5 & 1 & 2 & 7 & 2 & 63 \\
\hline
\end{tabular}

Table 3. Distribution of cases of human rabies.

\begin{tabular}{|c|c|c|}
\hline AGE & Staff & Percentage \\
\hline $0-19$ years & 49 & $77.8 \%$ \\
\hline$\geq 20$ years & 14 & $22.2 \%$ \\
\hline \multicolumn{3}{|l|}{ PROFESSION } \\
\hline Pre-school & 3 & $4.7 \%$ \\
\hline School & 30 & $47.8 \%$ \\
\hline Housewife & 13 & $20.6 \%$ \\
\hline Other & 17 & $26.9 \%$ \\
\hline \multicolumn{3}{|c|}{ LOCATION OF THE BITE } \\
\hline Lower limb & 35 & $55.6 \%$ \\
\hline Upper limb & 15 & $23.8 \%$ \\
\hline Multiple bites & 13 & $20.6 \%$ \\
\hline \multicolumn{3}{|c|}{ NATURE OF THE BITE } \\
\hline Superficial & 44 & $63.4 \%$ \\
\hline Deep & 19 & $36.6 \%$ \\
\hline \multicolumn{3}{|l|}{ LOCAL CARE } \\
\hline Yes & 8 & $12.7 \%$ \\
\hline No & 55 & $87.3 \%$ \\
\hline
\end{tabular}




\section{Continued}

\begin{tabular}{ccc}
\hline 21 - 30 days & 6 & $15 \%$ \\
$31-60$ days & 52 & $82.5 \%$ \\
$\geq 61$ days & 5 & $12.5 \%$ \\
DURATION BETWEEN FIRST SYMPTOMS & & \\
AND DEATH & 10 & $15.8 \%$ \\
$<3$ days & 46 & $73 \%$ \\
3 to 7 days & 7 & $11.2 \%$ \\
$>7$ days & & \\
BITING ANIMAL IN QUESTION & 61 & $97 \%$ \\
Dog & 1 & $1.5 \%$ \\
Cat & 1 & $1.5 \%$ \\
Unknown & & \\
ORIGIN OF THE ANIMAL & 45 & $71.4 \%$ \\
On the run & 18 & $38.6 \%$ \\
Shot & &
\end{tabular}

Other* maneuver, apprentice driver, carpenter, bricklayer, civil servant, artisans, farmer, gardener, merchant.

\section{Comments}

\subsection{Prevalence of Human Rabies Cases}

From January 2007 to December 2017, sixty-three (63) new cases of human rabies have been observed in Bamako on a population of 1,809,106 inhabitants [3], or an annual incidence of 0.37 per 100,000 inhabitants, which is more important than the prevalence of rage cases in all member countries of the bureau of experts on rage cases of the African continent (AfroREB) for 2008, which was 0.028 for 100,000 inhabitants [4].

These results exposed above do not reflect the true iceberg of rabies inoculation. They must be multiplied by at least 10 to give a realistic idea of this iceberg of rabies inoculation [2].

\subsection{Socio-Demographic Characteristics of the Victims}

Two age groups were particularly affected by death resulting from human rabies with a pre-dominance in the infection rate at age $20(80 \%)$ (Table 3). This is explained by the fact of lack of vigilance or provocation of dogs at this age range of infection.

Students and women in the home and their household maids were the occupations most affected, with respectively $47.5 \%$ and $27.5 \%$ (Table 3 ). This is explained by the frequent contact of these two groups with the animals in the homes.

All the patients have been exposed to the disease in the urban areas of Bamako. These patients were mostly domiciled in commune VI (17 patients or 
$42.5 \%$ ), followed by the commune I (11 patients $27.5 \%$ ) then at the same time as commune IV and commune V with 4 patients or $10 \%$ of the cases (Table 3 ).

\subsection{Epidemiological Aspects}

Seven patients (17.5\%) had consulted a health center and none had received a post exposure prophylaxis, according to the Essen Protocol of the WHO, in force in Mali (Table 3). These 7 patients claimed to have received the local health care (immediate washing of the wound with water and soap, rinsing and application of an antiseptic) which constitutes a crucial step in the care and support of people exposed to rabies infection (Table 3 ). The low rate of persons who benefited from the local health care after exposure reflects a lack of knowledge of the rabies disease and the treatment to be given after exposure. It is therefore appropriate to implement a program to raise the awareness of the populations, so that this stage is carried out in the case of exposure. In addition, the lack of immunization coverage among those who have consulted health caregivers might be due to the financial inaccessibility to a vaccine. Indeed, frequent shortages of the rabies vaccine are observed in the rabies center. This situation forced the patients to procure the vaccine in the private pharmacies where prices vary between 10,855 FCFA and 10,960 FCFA (US\$21.71 to US\$21.92).

The bites were located in $80 \%(32 / 40)$ of our patients at the level of the body limbs (upper and lower) including 57.5\% (23/40) of the cases only at the level of the lower limbs (Table 3). They were superficial in 58\% (23/40) of the cases, and deep in $42 \%$ of the cases, but all were assimilated to the category III depending on the distribution of the WHO regarding the nature of the bite (Table 3).

The first symptoms were observed 20 days after the bite and the patients died three days after the occurrence of the first symptoms (Table 3 ). The median time between the bite and the onset of the first symptoms (incubation period) was 43.5 days with the extremes ranging from 21 days to 103 days (Table 3 ). The median time between the appearance of the first signs and death was four days with extremes ranging from two to ten days (Table 3). For the biological confirmation of human rabies cases, no biopsy of brain tissue could be realized in post-mortem. It has not been possible because of the refusal of biopsies by parents for socio-cultural reasons. The diagnosis of human rabies in ante-mortem developed from the analysis of a sample of skin having proved its specificity and its sensitivity [5] could be of a considerable contribution in our context for the confirmation of cases, which is an essential step in the surveillance of the disease.

\subsection{Animal Concerned and Type of Exposure}

In $95 \%$ of the cases of human rabies the type of exposure was the bite in the same proportion (Table 3). On all the animals under investigation, 24 (60\%) were in flight (Table 3). The sixteen (16) other animals have been slaughtered and the dead bodies have not been sent to the competent services for analysis (Table 3). Indeed, the offending animal was the dog which remains the main 
vehicle of contamination for the vast majority of tropical countries where $95 \%$ of human infections are due to dog bites [1]. And that is a real public health problem or the purpose of the establishment of a good animal health policy based on a competent mastery of the canine ecology which would allow for better knowledge of the current density of the canine population in Bamako and to put in place programs to combat these enzootic or incursional zones of rabies cases.

\section{Conclusion}

This study has allowed us to see that the rage disease is an unknown and underestimated reality in Mali. The implementation of an efficient system of epidemiological surveillance is essential to measure its real impact and take appropriate action for its elimination.

\section{Acknowledgements}

Aïchatou Margueritte Diarra-MBA in Small and Medium Enterprise Management and Entrepreneurship-for the correction of the article.

\section{Conflicts of Interest}

The authors declare no conflicts of interest regarding the publication of this paper.

\section{References}

[1] Knobel, D.L., Cleaveland, S., Coleman, P.G., Fevre, E.M., Meltzer, M., Miranda, I., et al. (2005) Re-Evaluation of the Burden of Rabies in Africa and Asia. Bulletin of the World Health Organization, 83, 360-368.

[2] World Health Organization (2005) WHO Expert Committee on Rabies: First Report. World Health Organization, Geneva, Series of Technical Reports of WHO, No. 931. https://doi.org/10.1037/e459952004-001

[3] National Institute of Statistics of Mali (2011) Report of the 4th General Census of the Population of Mali, Bamako.

[4] Dodet, B., Adjogoua, E.V., Aguemon, A.R., et al. (2008) Fighting Collaborating Center in Africa: The Africa Expert Collaborating Center Office (AfroREB). Vaccine, 26, 6295-6258.

[5] Dacheux, L., Reynes, J.M., Buchy, P., Sivuth, O., Diop, B.M., Rousset, D., et al. (2008) A Reliable Diagnosis of Human Collaborating Center Based on Analysis of Skin Biopsy Specimens. CID, 47, 1410. https://doi.org/10.1086/592969 